\title{
INFLUENCE OF ATOMIC AND ELECTRONIC STRUCTURE OF A TIP ON STM IMAGES
}

\author{
L. JURCZYSZYN \\ Institute of Experimental Physics, University of Wrocław \\ Pl. M. Borna 9, 50-204 Wrocław, Poland \\ N. Mingo and F. Flores \\ Departamento de Fisica de la Materia Condensada, C-V, Facultad de Ciencias \\ Universidad Autonoma de Madrid \\ 28049 Madrid, Spain
}

Theoretical studies of the influence of electronic and atomic structure of a tip on scanning tunneling microscopy images are presented. Results are discussed for the scanning of $\mathrm{Al}(001)$ and $\mathrm{Al}(001)+\mathrm{Xe}$ surfaces performed within aluminum and nickel tips of a different geometry.

PACS numbers: $07.79 . \mathrm{Cz}, 61.16 . \mathrm{Ch}$

\section{Introduction}

This article presents some theoretical studies related to a general problem of interpretation of scanning tunneling microscopy (STM) results. More specifically, we have investigated the influence of electronic properties of a tip as well as its geometry on the multichannel tunneling of electrons in a tip-sample system.

In particular, we have discussed how the electronic and atomic structure of different tips could change the topography of STM images. This problem has been considered in detail for STM images of a single Xe atom adsorbed on an $\mathrm{Al}(001)$ surface as well as for those of a clean $\mathrm{Al}(001)$ surface; our calculations have been performed for $\mathrm{Al}$ and $\mathrm{Ni}$ tips of a different geometry.

\section{Model and method of calculation}

To calculate the tunneling current in a tip-sample system, we have used the non-equilibrium Green-function method developed by Keldysh [1]. This approach was used by Caroli et al. in theoretical investigations of the tunneling phenomena in microstructures $[2,3]$. Later it was also adopted for STM study $[4,5]$. A detailed description of this method is presented in [6]. It is shown there that for calculation 
of a tunneling current, the matrices of the Green functions and the density of states for the tip and the sample are needed.

In our study, calculations of the Green functions and the density of states of the sample have been done within the self-consistent LCAO approach, described in detail in [7]. On the other hand, the tip has been, described using the cluster-Bethe-lattice method: the top of a tip is represented by a cluster of a few atoms, while the influence of the rest of the tip is simulated by a Bethe lattice connected to this cluster (cf. Fig. 1).

a)

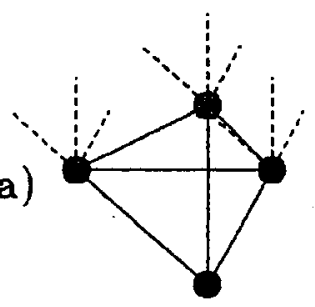

b)

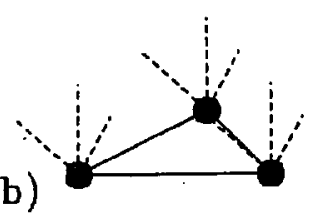

c)

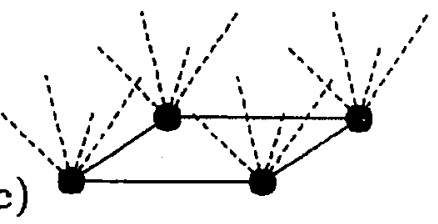

Fig. 1. Different geometries of the top part of a tip considered: sharp tip with a single atom located at the apex (a), and flat tips (i.e., without the apex atom) formed by three (b) or four (c) atoms.

Such a model enables us to consider different geometries of the top of a tip. We have assumed it has a form of pyramid with a single atom at the apex and three atoms at the basis (cf. Fig. 1a). For comparison, we have also considered two tips without the apex atom, i.e., with a flat top formed by three or four atoms (cf. Figs. $1 \mathrm{~b}$ and c, respectively). In all the cases shown in Fig. 1 the geometry of the tip is determined by the fcc structure of a considered metal (i.e., $\mathrm{Al}$ or $\mathrm{Ni}$ ), with distances between the atoms forming the tip equal to those in the bulk.

\section{3, Results and discussion}

\subsection{Al(001)+Xe surface}

First we shall discuss the influence of the tip properties on STM images of a single $\mathrm{Xe}$ adatom on $\mathrm{Al}(001)$ surface. The results obtained within the sharp aluminum tip (as in Fig. 1a) are shown in Fig. 2a, where the conductance evolution along the line passing above the adsorbed Xe atom is presented for different tip-sample distances $d$ (constant height mode for each $d$ is assumed). It follows that for large enough $d$ the protrusion of Xe adatom has a typical shape with one maximum located exactly above the adsorbed atom. For $d \approx 4.2 \AA$ the conductance-dependence curve becomes flat at the top, and for still smaller $d(d<4 \AA)$ it starts to exhibit a crater-like shape with a minimum located above the adatom.

This behavior is due to electronic properties of the aluminum tip. Indeed, Figs. $2 \mathrm{~b}-\mathrm{d}$ present evolution of the conductance resulting from the tunneling via $s, p_{z}$, and $p_{y}$ orbitals of the apex atom, respectively. As it can be seen, for greater tip-sample distances the main contribution to the total conductance comes from 


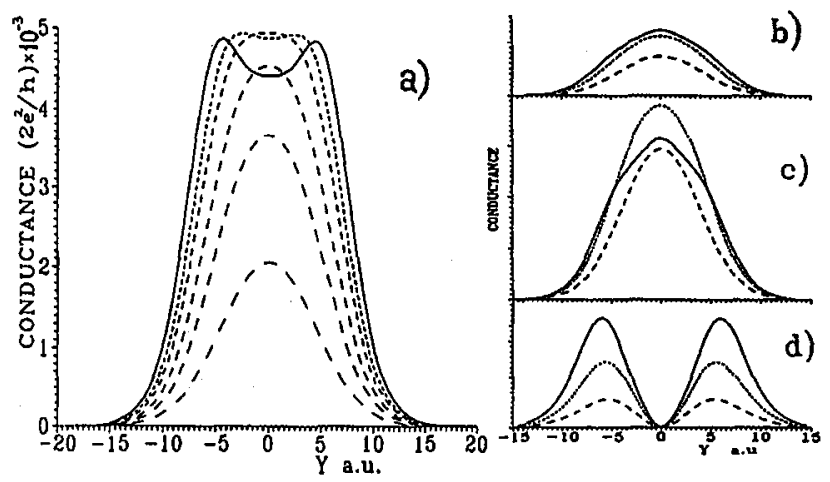

Fig. 2. (a) Evolution of the conductance along the line passing above $\mathrm{Xe}$ atom adsorbed on $\mathrm{Al}(001)$ surface and located at $y=0$, obtained within a sharp Al tip (as in Fig. 1a). Different curves correspond to different heights $d$ of the tip above Xe adatom: $d=3.1 \AA$ (solid curve), $d=3.48 \AA, d=3.76 \AA, d=4.2 \AA, d=4.6 \AA$, and $d=5.33 \AA$ (dashed curves with an increasing length of dash corresponding to an increasing value of $d$ ). (b), (c), and (d) represent the $s, p_{z}$, and $p_{x y}$ orbital contribution to the total conductance, respectively. Solid, dotted, and dashed curves correspond to $d=3.1 \AA, d=3.76 \AA$, and $d=4.6 \AA$, respectively.

the tunneling via $s$ and $p_{z}$ orbitals ( $p_{z}^{\prime}$ being perpendicular to the surface). Under such conditions the current flows mainly from the region located directly below the tip, thus the conductance reaches its maximum value just above the Xe adatom. On the other hand, $p_{y}$ and $p_{x}$ orbitals are parallel to the surface, so the tunneling via them takes place not from the region just below the tip, but rather from its surrounding. As a result, the conductance via $p_{x y}$ orbital has two maxima situated symmetrically with respect to the position of Xe atom (cf. Fig. 2d). Since tunneling via $p_{y}$ and $p_{x}$ orbitals becomes more effective for smaller tip-sample distances, this considerably influences the evolution of the total conductance and creates a crater-like shape (cf. Fig. 2a for $d<4 \AA$ ).

Figure 3 presents the corresponding results obtained within a flat aluminum tip with a triangle top (as in Fig. 1b). Three topmost atoms are equally active in electron tunneling, which leads to a considerably higher conductance and a broader Xe protrusion (cf. Fig. 3a). For smaller tip-sample distances, the conductance-dependence curve shape is strongly influenced by the atomic structure of the flat top of the tip - as a consequence, the image of a single Xe adatom has a complicated and untrue structure (cf. Fig. $3 \mathrm{~b}$ ). For greater $d$, when the tunneling takes place again mainly via $s$ and $p_{z}$ orbitals, the effect of the atomic structure of the tip becomes less important.

\subsection{Clean Al(001) surface}

First, we shall discuss the results obtained within a sharp aluminum tip (cf. Fig. 1a). Figure 4a presents evolution of the total conductance along the $\mathrm{Al}(001)$ surface (constant height mode) for the tip-sample distance $d=3.62 \AA$, but this result is typical also of greater distances. Under such conditions, tunneling 

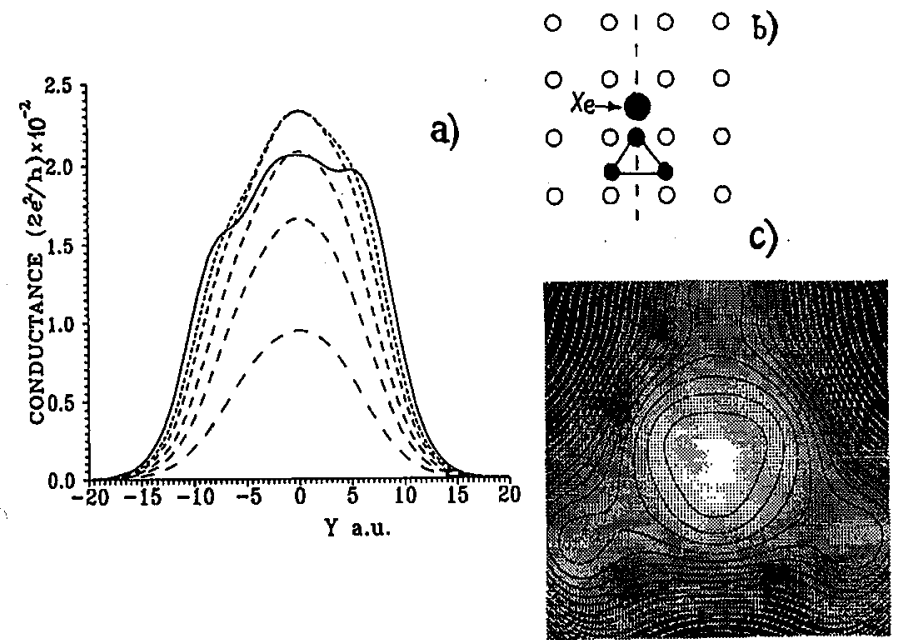

Fig. 3. (a) Evolution of the conductance along the line passing above Xe atom adsorbed on $\mathrm{Al}(001)$ surface, obtained within a flat Al tip with a triangle top (as in Fig. 1b). Different curves correspond to different heights $d$ of the tip above Xe adatom: $d=3.1 \AA$ (solid curve), $d=3.48 \AA, d=3.76 \AA, d=4.2 \AA, d=4.6 \AA$, and $d=5.33 \AA$ (dashed curves with an increasing length of dash corresponding to an increasing value of $d$ ). (b) Tip orientation with respect to the sample surface. Solid and open circles denote the positions of atoms from the top of the tip and from the surface, respectively, while the dashed line shows the direction of a tip displacement. (c) Image of the Xe adatom calculated for $d=3.48 \AA$.

via $s$ and $p_{z}$ orbitals of the apex atom takes place mainly from the region located directly below the tip, hence the $s$ and $p_{z}$ contributions to the total conductance reach maximum whenever the tip is located above a surface atom (cf. Figs. $4 \mathrm{~b}$ and c). Because the tunneling via $p_{x}$ and $p_{y}$ orbitals takes place not from the region located directly below the tip, but rather from its surrounding, the $p_{x y}$ contribution has maximum above the hollow points of the surface structure (cf. Fig. 4d). For greater tip-sample distances, however, the tunneling via $p_{x y}$ orbitals is not effective (10-16\% of the total conductance), so the evolution of the conductance along the surface is determined by $s$ and $p_{z}$ contributions. Therefore, the obtained image (cf. Fig. 4a) shows a normal topography (conductance has maxima above surface $\mathrm{Al}$ atoms).

Figure 5 presents the corresponding results for $d=2.34 \AA$. For such small distances, when the tip is above a surface atom, the $s$ and $p_{z}$ contributions quickly reach saturation, so they become higher above the hollow and bridge points than above the surface atoms (cf. Figs. 5b and c). Moreover, the $p_{x y}$ contributions are more important for smaller $d$ (about $78 \%$ of the total conductance) and they show maxima above the surface atoms as well as the hollow points (cf. Fig. 5d). The superposition of all the contributions gives now the image with an inverted topography (cf. Fig. 5a - the conductance has maxima above the hollow points). 
a)

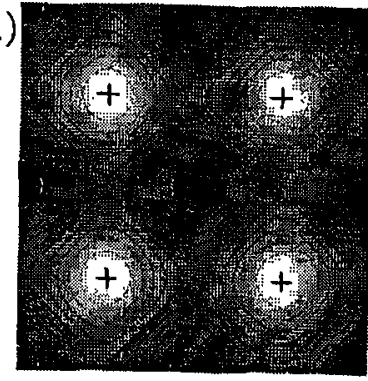

c)

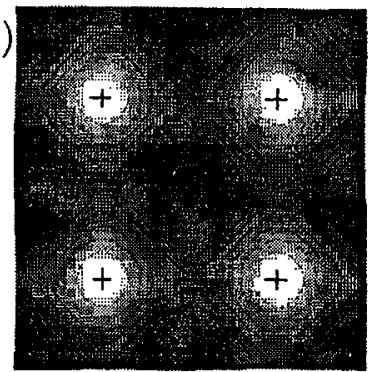

b)

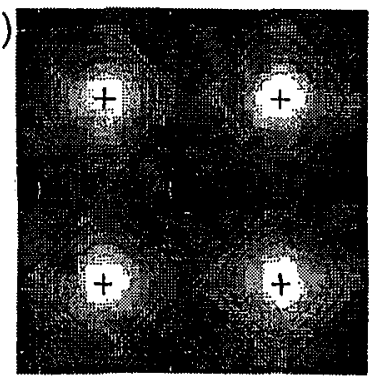

d)

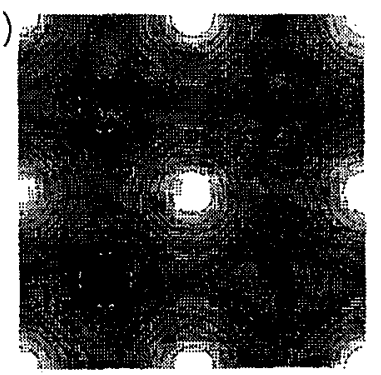

Fig. 4. (a) Image of a clean $\mathrm{Al}(001)$ surface (constant height mode), obtained within a sharp Al tip (as in Fig. 1a) for $d=3.62 \AA$. (b), (c), and (d) represent the conductance components connected with the tunneling via $s, p_{z}$, and $p_{x y}$ orbitals, respectively. Black crosses denote the positions of surface atoms.

a)

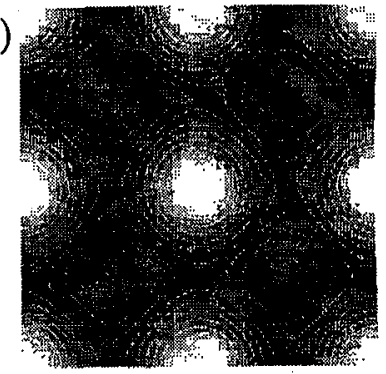

c)

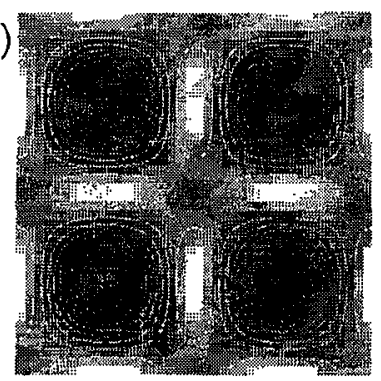

b)

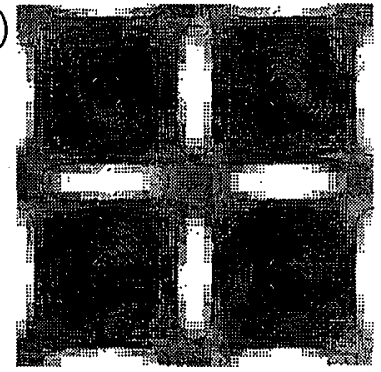

d)

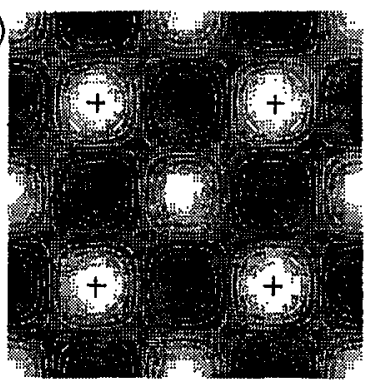

Fig. 5. The same as in Fig. 4, but for $d=2.34 \AA$. 
The case of a flat $\mathrm{Al}$ tip (i.e., without the apex atom) has been studied for the tip with a triangle and square top (cf. Fig. $1 \mathrm{~b}$ and c, respectively). The evolution of the conductance is determined now by the superimposed tunneling through $s, p_{z}$ and $p_{x y}$ orbitals of all the three or four atoms forming the top of a tip. Figure 7 presents the STM images calculated for $d=3.62 \AA$ (representative also for greater distances). Then the tunneling takes place mainly through $s$ and $p_{z}$ orbitals, from the regions located directly below the respective top atoms. The calculations have been performed for different orientations of the tip with respect to the surface structure, as it is schematically shown in Figs. 6a-c. It follows from the obtained

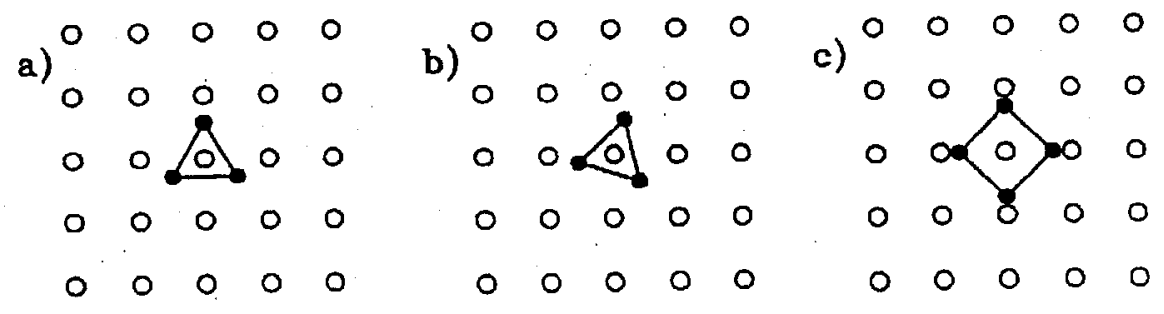

Fig. 6. Different orientations of a flat Al tip with respect to the surface atomic structure of $\mathrm{Al}(001)$. (a) and (b) correspond to the tip with a triangle top ((b) being rotated by 15 degrees as compared to (a)), while (c) represents the tip with a square top.
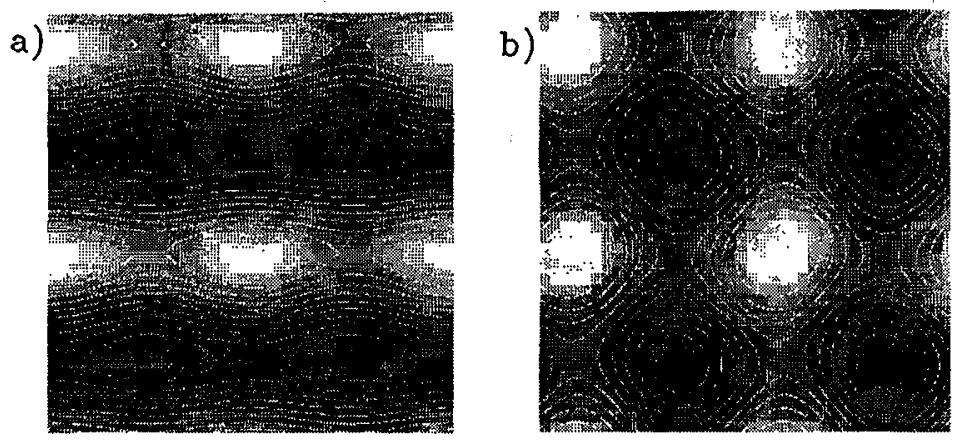

Fig. 7. Images of a clean $\mathrm{Al}(001)$ surface (constant height mode), obtained within a flat Al tip with a triangle top for $d=3.62 \AA$. (a) and (b) correspond to the tip orientations as in Fig. $6 a$ and $b$, respectively.

results that the tip with a triangle top, oriented as in Fig. 6a, gives the image with an untrue structure of rows (cf. Fig. 7a). But rotating this tip by 15 degrees (i.e., as in Fig. 6b) leads to the image with a structure of peaks reproducing the geometry and distances of the surface structure (cf. Fig. $7 \mathrm{~b}$ ). It should be noticed, however, that in both cases the positions of conductance maxima do not correspond to the true positions of surface atoms.

Figures 8 and 9 present the images of a clean $\mathrm{Al}(001)$ surface for a variable tip-sample distance $d$, obtained within a flat Al tip with a triangle and square top, 
a)

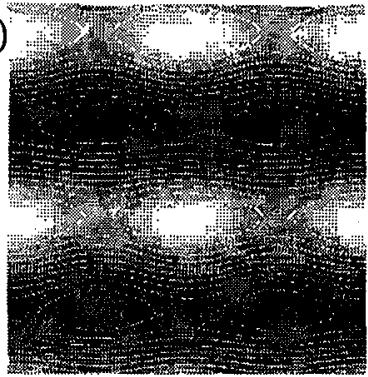

d)

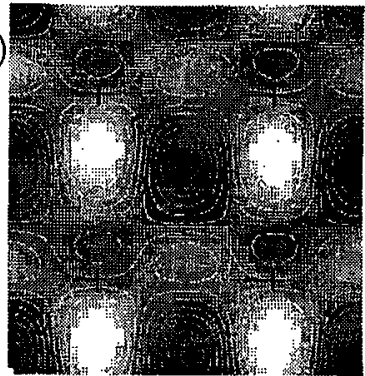

b)

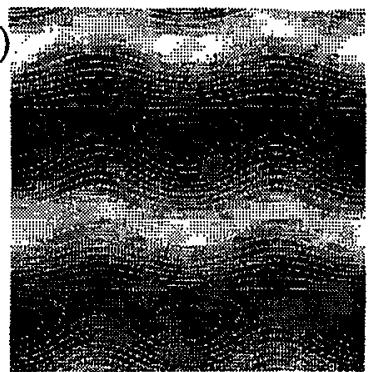

e)

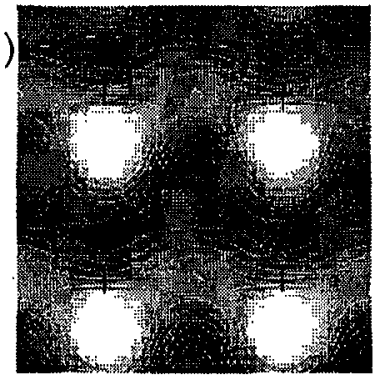

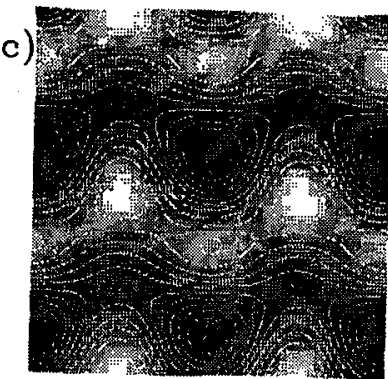

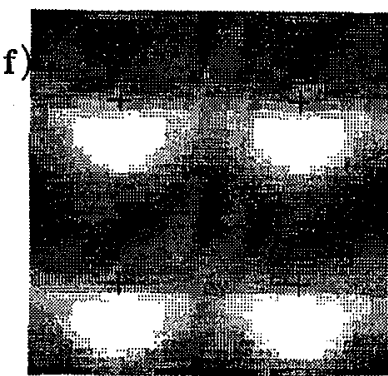

Fig. 8. Set of images of a clean $\mathrm{Al}(001)$ surface, obtained within a flat Al tip with a triangle top and oriented as in Fig. 6a, for a decreasing tip-sample distance $d$ : (a) $3.62 \AA$, (b) $3.13 \AA$, (c) $2.98 \AA$, (d) $2.84 \AA$, (e) $2.7 \AA$, and (f) $2.34 \AA$.

a)

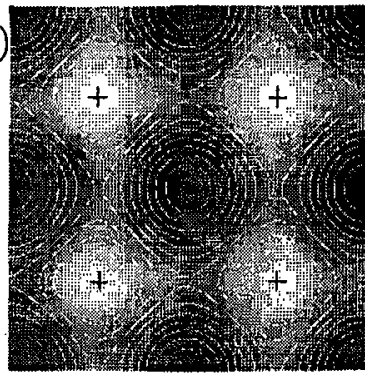

d)

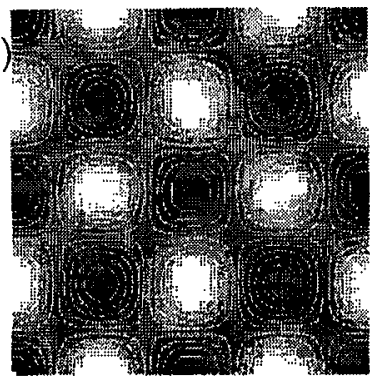

b)

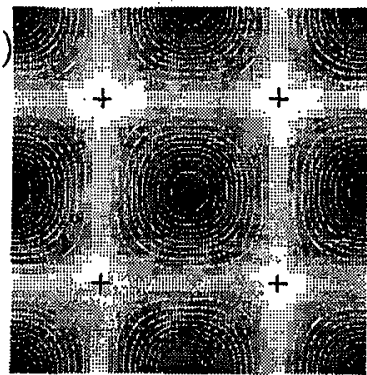

e)

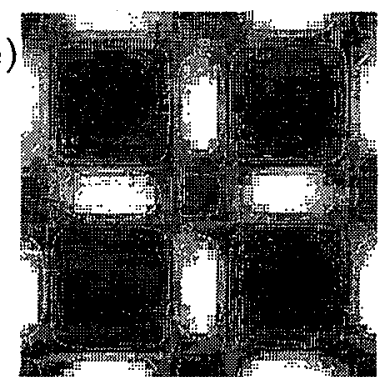

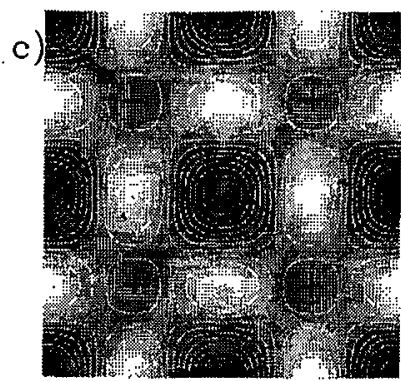

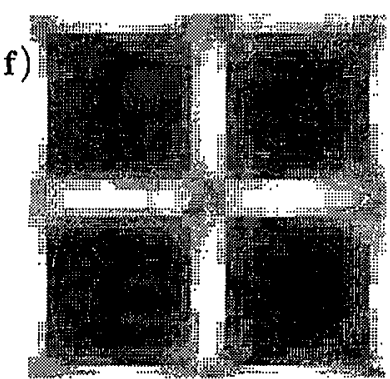

Fig. 9. Set of images of a clean $\mathrm{Al}(001)$ surface, obtained within a flat $\mathrm{Al}$ tip with a square top and oriented as in Fig. $6 \mathrm{c}$, for a decreasing tip-sample distance $d$ : (a) $3.62 \AA$, (b) $3.27 \AA$, (c) $3.13 \AA$, (d) $2.98 \AA$, (e) $2.7 \AA$, and (f) $2.34 \AA$. 
and oriented as in Fig. 6a and c, respectively. For $s$ and $p_{z}$ orbitals, tunneling from the region located below the respective tip atom becomes - with decreasing $d-$ less effective than from its surrounding. This effect, as well as the more significant tunneling via $p_{x y}$ orbitals, cause important changes of the obtained images. As a result, they have complicated topographies, very different from a clean $\mathrm{Al}(001)$ surface atomic structure.

To study the influence of $d$ orbitals of atoms forming the tip on STM images, calculations have been also performed for a triangle flat tip made of $\mathrm{Ni}$ atoms. The images obtained for $d=3.62 \AA$ are shown in Fig. 10 for two different orientations (as in Figs. $6 \mathrm{a}$ and b, respectively). For such a distance almost $85 \%$ of the current flows via $s$ orbitals of $\mathrm{Ni}$ atoms. In contrast to the $\mathrm{Al}$ tip, however, the $s$ orbitals of $\mathrm{Ni}$ atoms do not contribute to the current only from the regions located directly below these atoms - this is due to a greater radius of $s$ orbital of $\mathrm{Ni}$ atom as compared to $\mathrm{Al}$ atom. As a consequence, the corrugation implied by $s$ orbitals is very small, and the topography of the images shown in Fig. 10 is caused mainly by $d$ and $p_{z}$ orbitals of the topmost atoms. The tip orientation as in Fig. 6a gives,

a)

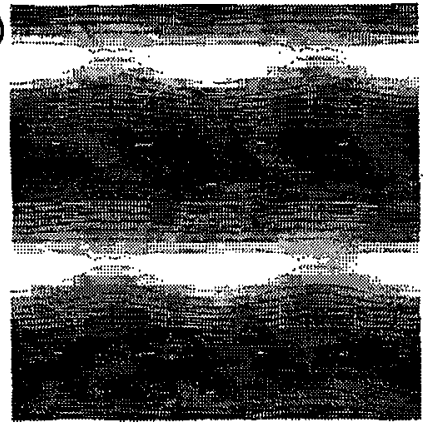

b)

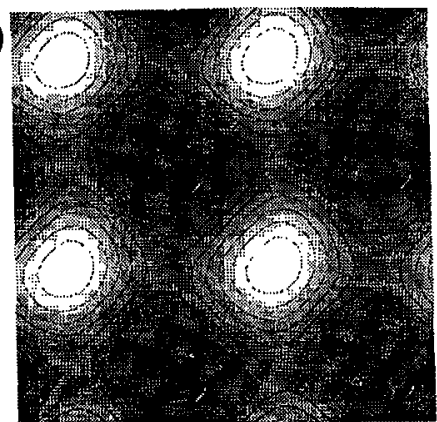

Fig. 10. The same as in Fig. 7, but for a Ni tip.

a)

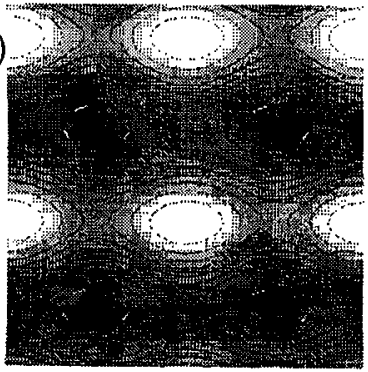

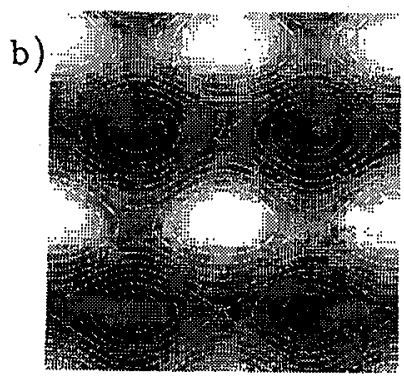

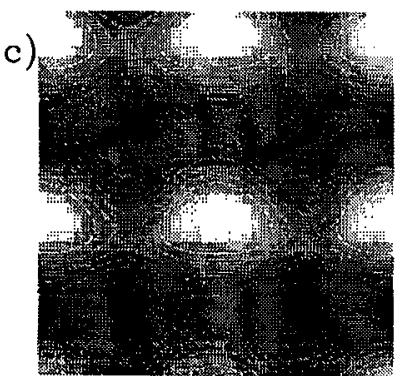

Fig. 11. Set of images of a clean $\mathrm{Al}(001)$ surface, obtained within a flat $\mathrm{Ni}$ tip with a triangle top and oriented as in Fig. $6 \mathrm{a}$, for a decreasing tip-sample distance $d$ : (a) $3.13 \AA$, (b) $2.7 \AA$, and (c) $2.34 \AA$. 
like in the case of Al tip, an image with the structure of untrue rows, while the tip rotation by 15 degrees changes completely this topography (cf. Fig. 10b).

For smaller distances (cf. Fig. 11), the tunneling current is dominated entirely by the contribution of $d$ orbitals. Because of the strong localization of these orbitals, their current components come mainly from the regions located directly below the respective tip atoms. This explains the topographies of the obtained images (cf. Figs. 11a-c). As it can be seen - contrary to the case of Al tip decreasing the tip-sample distance does not lead to any important changes of the STM images.

\section{Conclusions}

Theoretical studies presented in this paper indicate that the electronic and atomic properties of a tip considerably influence STM images. This effect seems to be important for the investigation of structural properties of sample surfaces within STM technique.

We have found that flat tips (i.e., without apex atom) give images of an $\mathrm{Al}(001)$ surface with an atomic resolution. On the other hand, their topographies could have untrue structures and features, which is caused by the geometry of the flat top of a tip, its orientation with the respect to the surface structure, and electronic properties of the atoms forming the tip. The $d$ states of the tip atoms give an important contribution to electron tunneling in the tip-sample system, especially for small distances; a strong localization of $d$ orbitals results in a local character of tunneling through them, so it takes place mostly from the regions located directly below the tip atoms.

\section{Acknowledgments}

One of the authors (L.J.) acknowledges a support of the University of Wroctaw within grant 2016/W/IFD/96.

\section{References}

[1] L.V. Keldysh, Zh. Eksp. Teor. Phys. 47, 1515 (1964) [Sov. Phys. JETP 20, 1018 (1965)].

[2] C. Caroli, R. Combescot, P. Nozieres, D. Saint-James, J. Phys. C 4, 916 (1971).

[3] C. Caroli, R. Combescot, P. Nozieres, D. Saint-James, J. Phys. C 5, 21 (1972).

[4] A. Martin-Rodero, F. Flores, N.H. March, Phys. Rev. B 38, 10047. (1988).

[5] J. Ferrer, A. Martin-Rodero, F. Flores, Phys. Rev. B 38, 10113 (1988).

[6] N. Mingo, L. Jurczyszyn, F.J. Garcia-Vidal, R. Saiz-Pardo, P.L. de Andres, F. Flores, S.Y. Wu, W. More, Phys. Rev. B 54, 2225 (1996).

[7] F.J. Garcia-Vidal, A. Martin-Rodero, F. Flores, J. Ortega, R. Perez, Phys. Rev. $B$ 44, 11412 (1991). 